\title{
Exogenous visual attention prolongs perceived duration
}

\author{
Tanja Seifried • Rolf Ulrich
}

Published online: 20 November 2010

(C) Psychonomic Society, Inc. 2010

\begin{abstract}
Previous studies have shown that spatial attention prolongs the perceived duration of visual stimuli. Chen and O'Neill (Perception \& Psychophysics, 63, 1229-1238, 2001) reported, however, the reversed result and thus challenged the generality of this attention effect. We therefore reinvestigated the influence of spatial attention on the perceived duration of visual stimuli in various experimental settings. In five experiments, perceived duration was longer for validly cued stimuli; thus, spatial attention was found to prolong perceived duration. In a further experiment, the specific conditions were such that neither the attention effect nor the reversed pattern, as found by Chen and O'Neill, emerged. Although we cannot provide a conclusive account of why Chen and O'Neill observed the reversed attention effect, the present results reinforce the generality of the spatial attention effect on perceived duration but also make advisable a systematic examination of the factors that might modulate this effect.
\end{abstract}

Keywords Spatial attention · Perceived duration .

Exogenous cuing

Humans perceive the temporal succession of two events, as well as the duration of these events. We call this time perception, although it is not analogous to other forms of perception, or as Woodrow (1951) put it, "time is not a

T. Seifried $(\bowtie) \cdot R$. Ulrich

Psychologisches Institut, Universität Tübingen,

Friedrichstrasse 21,

72072, Tübingen, Germany

e-mail: tanja.seifried@uni-tuebingen.de thing that, like an apple, may be perceived" (p. 1235). Even though each stimulus has both non-temporal and temporal features, the temporal features are rather indirect, whereas most non-temporal features are more direct and tangible. For visual stimuli, for example, color mainly depends on wave length, and we have specialized color receptors to perceive this stimulus feature. In contrast, time cannot be reduced to such a concrete characteristic as wave length, and presently no temporal sensory receptors can be identified (see Grondin, 2001). Consequently, time perception and, especially, the perceived duration of a temporal interval are always linked to other features of the stimulus by which it is represented and to the factors of the situation in which it is perceived. Knowledge about the nature of these factors and the way in which they affect perceived duration is of great relevance for models of the functionality of time perception. In the present article therefore, we try to add to this body of knowledge.

A variety of stimulus features are known to influence time perception and, especially, perceived duration. It is crucial, for example, whether the temporal interval is filled or empty (Grondin, 1993; Thomas \& Brown, 1974) and in which sensory modality the interval is represented (Wearden, Edwards, Fakhri, \& Percival, 1998). Further relevant features are intensity (Brigner, 1986), size of the stimuli (Thomas \& Cantor, 1975), number of the stimuli (Xuan, Zhang, He, \& Chen, 2007), motion (Brown, 1995), and stimulus oddity (Tse, Intriligator, Rivest, \& Cavanagh, 2004). Tse et al., for example, employed an oddball paradigm, in which an odd comparison stimulus that varied in duration was randomly interspersed within a series of standard stimuli with a constant duration. These authors found that the 
odd stimulus' duration was overestimated; that is, it was perceived to be longer. This overestimation of odd, infrequent stimuli has been attributed to an increase in arousal (Ulrich, Nitschke, \& Rammsayer, 2006) elicited by odd stimuli.

Arousal is also one of the situational factors known to influence perceived duration. Penton-Voak, Edwards, Percival, and Wearden (1996), for example, presented auditory clicks before the temporal interval to be judged and, thereby, increased arousal (see also Treisman, Faulkner, Naish, \& Brogan, 1990). This led to an increased perceived duration of the stimuli that were experienced under higher arousal. The situational factor with the highest impact on perceived duration, however, is most likely attention.

An extensive number of studies (e.g., Brown, 1997; Hemmes, Brown, \& Kladopoulos, 2004; Macar, Grondin, \& Casini, 1994; Rammsayer \& Ulrich, 2005) have shown that detracting attention from a temporal task affects time perception and diminishes perceived duration in particular. The paradigm in these studies is usually a dual-task paradigm, which requires participants to perform a duration judgment task and, concurrently, a non-temporal task. In an experiment by Brown (1997), for example, participants produced a duration series of 2- or 5-s intervals by pressing a key repeatedly. Either participants performed this temporal task exclusively, or they were to perform a non-temporal task (e.g., mental arithmetic) concurrently. This nontemporal task could be either easy or difficult. The results showed that produced durations increased from no concurrent task to easy concurrent task to difficult concurrent task. Since longer productions indicate decreased perceived duration (see Zakay \& Block, 1997), the obtained pattern of results therefore supports the notion that a concurrent non-temporal task detracts attention from time perception and, thereby, diminishes perceived duration.

Whereas, in these studies, participants divided their attention between two tasks, a more recent research branch has investigated the effect of directed attention to specific locations in the visual field (Chen \& O'Neill, 2001; Enns, Brehaut, \& Shore, 1999; Mattes \& Ulrich, 1998; Yeshurun \& Marom, 2008). These studies showed that such spatial attention prolongs perceived duration. Mattes and Ulrich, for example, used endogenous arrow cues to direct attention in a traditional cuing paradigm (e.g., Posner, Nissen, \& Ogden, 1978). First, an arrow pointed to either the left or the right side of a fixation cross. Then a standard stimulus, which had the same duration throughout the experiment, was presented in the middle of the screen. Finally, a comparison stimulus with varying duration appeared on either the validly cued or the invalidly cued side of the display. Participants had to judge the comparison duration as either "shorter" or "longer" than the standard duration. The results showed that perceived duration tended to be longer for validly cued comparison stimuli than for invalidly cued ones. Thus, spatial attention prolonged perceived duration.

Spatial attention can be divided into endogenous and exogenous attention. The former is the rather slow, conscious, and conceptually driven component. The latter is rather fast, automatic, and stimulus driven (cf. Nakayama \& Mackeben, 1989). ${ }^{1}$ Mattes and Ulrich (1998), as well as Enns et al. (1999), directed spatial attention with central arrow cues and, thereby, manipulated endogenous attention. Exogenous attention has, however, also been shown to prolong perceived duration (Yeshurun \& Marom, 2008). Yeshurun and Marom assessed the perceived duration of an attended visual stimulus in comparison with a neutral one. Each stimulus were shown after the other on each trial; their order was random. Both stimuli were cued, either by a neutral cue that did not indicate the stimulus' location, but only its onset, or by a peripheral bar cue that indicated onset as well as location. This cue was short $(50 \mathrm{~ms})$ and was supposed to capture attention automatically and exogenously (Müller \& Rabbitt, 1989; Posner, 1980). Participants judged which of the two stimuli had the longer duration. Yeshurun and Marom found that the perceived duration of the attended stimuli was longer than that of the unattended ones and could, thereby, show that not only endogenous, but also exogenous spatial attention prolongs perceived duration. $^{2}$

The studies by Enns et al. (1999), Mattes and Ulrich (1998), and Yeshurun and Marom (2008) unequivocally demonstrate that visual spatial attention, be it endogenous or exogenous, prolongs perceived duration. However, there is one study that has shown exactly the opposite effect. Chen and O'Neill (2001) presented a cue that was followed by the letter $\mathrm{X}$ or $\mathrm{O}$ at the cued or an uncued location. Afterward, participants judged the letter's duration as "short," "medium," or "long." As a result, the duration was judged as shorter for validly cued letters. This result was found for exogenous luminance cues, as well as for endogenous arrow cues. Hence, in contrast to the studies described above, Chen and O'Neill found spatial attention to diminish perceived duration. In the remainder of this

\footnotetext{
${ }^{1}$ Nakayama and Mackeben (1989) termed the two components sustained and transient attention, respectively.

${ }^{2}$ Note that in Experiment 4 of Yeshurun and Marom (2008) a validly, as well as an invalidly, cued stimulus were compared with a neutrally cued one. Yeshurun and Marom found that perceived duration was prolonged for the validly cued stimulus but was unchanged for the invalidly cued one. This finding suggests an increase in perceived duration for attended stimuli, and not a decrease in perceived duration for unattended ones.
} 
article, we will refer to this finding as the reversed attention effect. ${ }^{3}$

Especially in the light of the most recent finding by Yeshurun and Marom (2008), the reversed effect of spatial attention on perceived duration found by Chen and O'Neill (2001) requires reinvestigation. Whereas there are two studies supporting the prolonging effect of endogenous attention, there is a tie for exogenous attention. Yeshurun and Marom have shown that it prolongs perceived duration, whereas Chen and O'Neill have shown that it diminishes perceived duration. Consequently, we want to shed some light on the opposing results of exogenous attention observed by Yeshurun and Marom and Chen and O'Neill. Specifically, we report the results of several experiments that were designed to isolate certain procedural characteristics that might have contributed to the opposing results reported by Chen and O'Neill. Moreover, we seek to reanalyze the effect of exogenous spatial attention on perceived duration with a different cuing setup and a different psychophysical measurement procedure, in order to evaluate the robustness of this effect. As was mentioned above, in order to inform models on time perception, it is essential to draw a consistent picture about the factors that affect perceived duration.

Specifically, in Experiments 1 and 2, we employed a simple cuing paradigm with a peripheral luminance cue to direct exogenous attention in the visual field. Experiment 1 challenges the account by Chen and O'Neill (2001) that the

\footnotetext{
${ }^{3}$ We recently replicated the effect reported by Chen and O'Neill (2001) in an experimental setting that was similar to the one used in their study. Therefore, we do not question the reality of the effect reported by these authors. This replication, however, is not included in this article, because here we focus on clarifying the reversed attention effect observed by Chen and O'Neill. Nevertheless, we provide here some methodological details about this experiment so that the reader can incorporate it into his understanding of the effect of visual attention on perceived duration. Essentially, this experiment was very similar to Experiment 3, with the following exceptions. The orientation of the position frames (horizontal or vertical) was varied over participants. Only one standard duration $(300 \mathrm{~ms})$ was used, and no adaptive procedure but a method of constant stimuli determined the duration of the comparison stimuli. The cue had a duration of $100 \mathrm{~ms}$, and the interval between cue offset and comparison stimulus onset was $200 \mathrm{~ms}$ long. The comparison stimulus itself was a gray square that completely filled the end of the frame in which it was presented; that is, the cue and comparison overlapped spatially, at least partially. The probability for a validly cued comparison stimulus was $75 \%$. The probability for an invalid same and an invalid different presentation was $12.5 \%$ each. That is, the comparison stimulus never appeared at the far position of the frames. An ANOVA with the factor of validity (valid vs. invalid same, or invalid different) showed a significant effect, $F(1,58)=4.59, p=.031$.Constant error $(13 \mathrm{~ms})$ was higher in the valid condition than in both invalid conditions taken together, as a Helmert contrast showed, $F(1,29)=5.21, p=.030$. That is, validly cued stimuli were judged to be shorter than invalidly cued ones.
}

amount of processing load modulates the effect of spatial attention on perceived duration. In Experiments 2 and 3, we assessed whether the setup of the cuing display might have some influence on the attention effect on perceived duration. Then, in Experiment 4, we investigated how the measurement method of perceived duration would affect the attention effect, and in Experiment 5, we examined the role of the type of cue. Finally, in Experiment 6, we employed an equality judgment and could, thereby, exclude response bias as an alternative explanation for the attention effect.

\section{Experiment 1}

In one experiment of Chen and O'Neill (2001), exogenous attention decreased perceived duration; in another one, however, exogenous attention prolonged perceived duration, just as in the study by Yeshurun and Marom (2008). The important difference between the two experiments by Chen and O'Neill that yielded such different results was whether the duration judgment was the primary and sole task or whether it was a secondary task. In the latter case, participants first had to identify the temporal stimulus as an $\mathrm{X}$ or an O. Only then, after this letter discrimination task, did they have to perform a duration judgment task - that is, to judge the duration of letter presentation as being "short," "medium," or "long." Chen and O'Neill concluded that it was this difference in processing load that led to the dissociating influence of exogenous attention on perceived duration.

When letter discrimination was the primary task, processing load was high, since the letters had to be identified and judged as being an X or an O. Under such high-load conditions, participants supposedly did not have enough resources left to process onset and offset of the stimulus adequately. Rather, they might have based their duration judgment on the perceived brightness of the stimuli. Since an attended stimulus appears brighter (e.g., Bashinski \& Bacharach, 1980), this would have led to prolonged perceived duration of the attended stimuli. In the condition in which participants could perform duration judgment as a primary task, Chen and O'Neill (2001) assumed that participants were less affected by the brightness of the stimuli, but more by their onset and offset. This means that participants tended to rely more on temporal cues than on the perceived intensity of the stimulus. Chen and O'Neill argued that the onset, as well as the offset, of attended stimuli are known to be detected earlier, so that invalidly cued stimuli would be detected later and would extinguish later. The authors further supposed that participants put more weight on the offset of a stimulus, so that the perceived duration of invalidly 
cued stimuli would be longer than the perceived duration of validly cued ones.

Although the brightness account might contribute to the prolonging effect of attention on perceived duration, the account for the condition with duration judgment as a primary task is speculative. First, Chen and O'Neill (2001) themselves noted that it is unclear under which conditions participants might put more weight on the offset of a stimulus. Second, Rolke, Ulrich, and Bausenhart (2006) showed that attention exactly does not speed the detection of stimulus offset but, rather, delays it. As a consequence of these considerations, it seems reasonable to put the processing load explanation of Chen and O'Neill to another test.

Thus, in Experiment 1, we varied spatial exogenous attention in a standard cuing paradigm with a peripheral luminance cue (see Fig. 1) and assessed perceived duration with a pair comparison method. The standard stimulus was always presented first and at fixation. To the left and to the right of fixation were two squared position frames. After the standard, a cue consisting of a brief whitening of one of the two frames (cf. Müller \& Rabbitt, 1989; Posner \& Cohen, 1984) was presented. Afterward, the comparison stimulus, a letter, appeared either in the cued or in the uncued frame. One group of participants had duration judgment as their sole task, whereas the other group had letter discrimination as a first task and was afterward prompted to judge the duration of the presented letter. According to Chen and O'Neill (2001), we should find the usual attention effect - that is, longer perceived duration for validly cued stimuli only for the second group of participants and the reversed effect for the first group. We employed two different standard durations, 100 and $300 \mathrm{~ms}$. Since interactions between standard duration and manipulated stimulus features have been reported in the literature (Long \& Beaton, 1980; Mattes \& Ulrich, 1998; Thomas \& Cantor, 1975), such a variation could provide additional information.

Method

\section{Participants}

Twenty-one female and 3 male students participated in this experiment. Their ages ranged from 20 to 36 years $(M=24.9$, $S D=4.0)$. All but 2 participants were right-handed, and all
Fig. 1 The trial sequence in Experiment 1: Validly cued trial from the condition with letter discrimination with an $\mathrm{X}$ as comparison stimulus

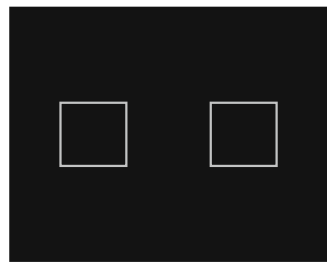

$1,000 \mathrm{~ms}$

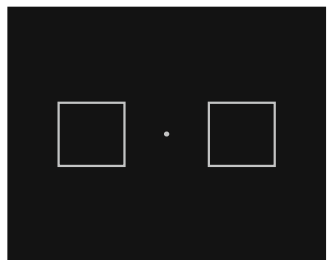

$1,000 \mathrm{~ms}$

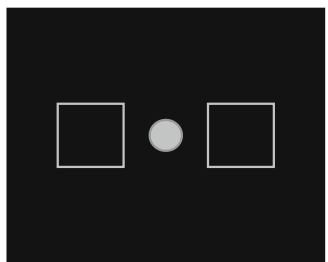

Standard duration

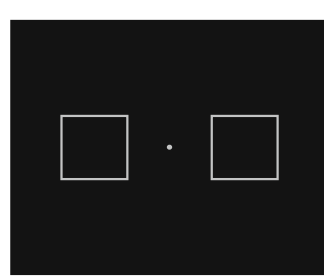

$1,000 \mathrm{~ms}$

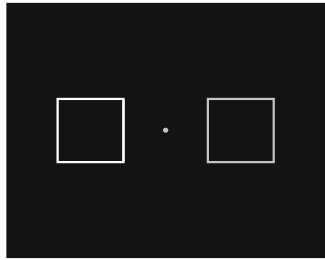

Cue: $53 \mathrm{~ms}$

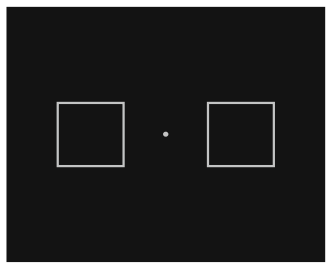

$53 \mathrm{~ms}$

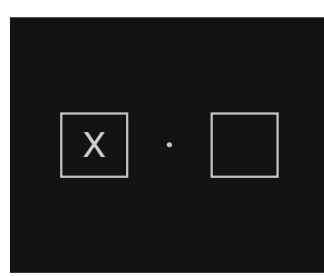

Comparison duration

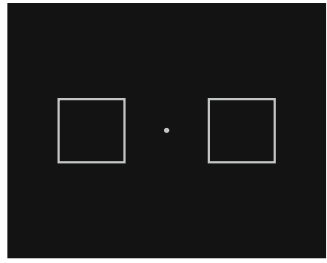

Until response

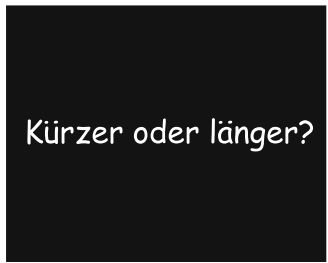

Response prompt

Time 
had normal or corrected-to-normal vision. They were told that the experiment was about time perception but were left naive as to the hypotheses of the experiment. Each student took part in one experimental session consisting of two parts and received $€ 9.5$ or course credit.

\section{Stimuli and apparatus}

The experiment was run in a sound-attenuated, dimly illuminated room. A PC controlled the experimental procedure and collected the participants' responses. The left and right arrow keys of a standard German keyboard served as the response keys for the letter discrimination task. The left and the right shift keys of the keyboard served as response keys for the duration judgment task. The experiment was programmed in Matlab ${ }^{\circledR}$ in conjunction with the Psychophysics Toolbox 3 (Brainard, 1997; Pelli, 1997). The position frames, as well as the fixation dot and the standard and comparison stimuli, were gray with a luminous intensity of $30 \mathrm{~cd} / \mathrm{m}^{2}$ and were presented on a black background $\left(<1 \mathrm{~cd} / \mathrm{m}^{2}\right)$. The cue was an intensity change of one of the position frames from gray to bright white $\left(100 \mathrm{~cd} / \mathrm{m}^{2}\right)$. The inner contours of the squared position frames were separated by $2.53^{\circ}$, and the thickness of the frames was $10.5 \mathrm{arcmin}$. The squared frames were located to the left and to the right of the screen center, with a distance of $5.7^{\circ}$ between them (this equals an eccentricity of $4.29^{\circ}$ ). The fixation dot had a diameter of 9.6 arcmin. The standard stimulus was a gray filled disk with a diameter of $1.4^{\circ}$. It appeared at the center of the screen, superimposed on the fixation point. The comparison stimulus was either the letter $\mathrm{X}$ or the letter $\mathrm{O}$. The letters had approximately the same size as the standard stimulus $\left(\mathrm{X}, 1.4^{\circ} \times 1.52^{\circ} ; \mathrm{O}, 1.4^{\circ} \times 1.51^{\circ}\right)$; the width was identical, but the height had to be adjusted to avoid distortions. The font type was Arial.

\section{Procedure}

Figure 1 shows the trial sequence for a validly cued trial with an $\mathrm{X}$ as the comparison stimulus. A trial started with the presentation of the position frames for $1,000 \mathrm{~ms}$. The frames, together with the fixation dot, were shown for another 1,000 ms before the standard was superimposed on the fixation dot. Depending on the standard duration of the experimental part, the standard was presented for either 100 or $300 \mathrm{~ms}$. Another presentation of the position frames for $1,000 \mathrm{~ms}$ was then followed by the cue. The cue was a brightening of the position frame on the left or the right side. This brightening lasted for $53 \mathrm{~ms}$, and the cuecomparison interval had the same duration. Thus, eye movements could not take place during the direction of attention; Mayfrank, Kimming, and Fischer (1987) sug- gested that it takes about $250 \mathrm{~ms}$ for a saccade to occur. The comparison stimulus could be either an $\mathrm{X}$ or an $\mathrm{O}$, which appeared on the same side as the cue (validly cued trials) or on the opposite side (invalidly cued trials). Whereas the duration of the standard stimulus was constant throughout one experimental part, the comparison duration varied from trial to trial. It was shorter than, equal to, or longer than the standard duration. After the presentation of the comparison stimulus, the trial proceeded differently for participants in the group with letter discrimination and for participants in the group without letter discrimination.

In the condition without letter discrimination, the trial sequence continued with the position frames for $1,000 \mathrm{~ms}$, and then a response prompt was shown. Participants were to judge the duration of the comparison stimulus as either "shorter" or "longer" than the standard stimulus by pressing the left or the right shift key, respectively.

In the condition with letter discrimination, the trial sequence also continued with the position frames. However, participants were required to make a speeded letter discrimination and to press the left arrow key for an $\mathrm{X}$ and the right arrow key for an $\mathrm{O}$. The position frames were replaced by the response prompt for the duration judgment task after a minimum of $1,000 \mathrm{~ms}$, after the participants' response, or after a maximum of 3,000 ms. Participants then were to judge the duration of the comparison stimulus as either "shorter" or "longer" than the standard stimulus by pressing the left or the right shift key, respectively. After the duration judgment, the screen turned black for an intertrial interval (ITI) of 1,000 ms for both groups.

Participants in both groups were told to fixate the center of the screen throughout the whole experiment and were supported in this requisition by the use of a chinrest. The importance of fixation and refraining from eye movements was emphasized several times throughout the experiment. Furthermore, participants were informed that the brightening of the position frame did not indicate on which side the comparison would appear - that is, the probability of validly and invalidly cued trials was $50 \%$ each - and that they should just fixate the screen center.

The complete experiment was divided into two parts, and each part employed a different standard duration. Each participant served in the two parts, which were performed on the same day. Half of the participants started with the 100-ms standard, and the other half with the 300-ms standard. The change of the standard duration after the first experimental part was announced to the participants after they had taken a short rest. Each experimental part comprised 2 (valid vs. invalid) $\times 2$ (cue left vs. cue right) $\times 2(\mathrm{X}$ vs. $\mathrm{O}) \times 2$ (upper run vs. lower run) $\times 13=208$ trials. It started with 32 practice trials, and then participants completed four experimental blocks of 52 trials. 
The duration of the comparison stimulus was changed according to a weighted up-down procedure (Kaernbach, 1991). This procedure estimates the 25 th (i.e., $x_{.25}$ ) and the 75th (i.e., $x_{.75}$ ) percentiles of the psychometric function in each experimental condition. These two points are the two corresponding comparison durations for which the participant responded "longer" on $25 \%$ and $75 \%$ of the trials, respectively. For the $100-\mathrm{ms}$ standard, the upper run started with a comparison duration of $126.7 \mathrm{~ms}$, and the lower run with $73.3 \mathrm{~ms}$. The starting values for the $300-\mathrm{ms}$ standard were 380 and $220 \mathrm{~ms}$, respectively. For the 100-ms standard, the comparison duration was increased by $6.7 \mathrm{~ms}$ for a "shorter" response and was decreased by $20 \mathrm{~ms}$ for a "longer" response when the 25 th percentile was estimated. The step sizes were reversed for the estimation of the 75th percentile. For the $300-\mathrm{ms}$ standard, the corresponding step sizes were 20 and $60 \mathrm{~ms}$. This procedure was employed for each of the four conditions per group ( 2 standard durations $\times 2$ validities). Since standard duration was blocked per experimental part, this resulted in four randomly interleaved runs per experimental part.

A maximum likelihood estimation procedure was used to estimate the point of subjective equality (PSE) and the difference limen (DL) for each participant in each of the four conditions. This procedure employs a logistic psychometric function (cf. Bush, 1963),

$P\left(x_{i}\right)=\frac{1}{1+e^{\frac{P S E-x_{i}}{0.91 \cdot D L}}}$

that associates the duration of the comparison stimulus $\mathrm{x}_{i}$ with the probability $P$ of the response "longer" (for further details, see Lapid, Ulrich, \& Rammsayer, 2008; see also Ulrich, 2010). The PSE is defined as $\mathrm{x}_{.50}$-that is, the comparison duration for which a participant responded "longer" with a probability of .50 . When a comparison stimulus was presented for this duration, the participant perceived it to be equally long as the standard stimulus. The DL is defined as $\left(\mathrm{x}_{.75}-\mathrm{x}_{.25}\right) / 2$ and refers to the just noticeable difference between two durations. From the PSE, the constant error (CE = PSE - standard) was computed. A negative (positive) CE or a lower (higher) PSE, respectively, indicates that a lower (higher) duration than the standard duration was needed in order for the comparison to be perceived as equally long as the standard.

This experiment factorially combined the two withinsubjects factors of standard duration (100 vs. $300 \mathrm{~ms})$ and validity (valid vs. invalid), as well as the between-subjects factor of letter discrimination (with vs. without). Reaction time (RT), CE, and DL were analyzed as dependent variables.

\section{Results and discussion}

The data of 1 participant were excluded from data analysis because his letter discrimination was at chance level. He was replaced by another participant. RT included only trials on which participants had responded correctly. Furthermore we excluded outlier trials that expressed RTs larger or smaller than $M \pm 3 \cdot S D$ (3 sigma rule; over all the trials in each condition). Altogether, we had to discard $5.9 \%$ of all the trials for the calculation of RT. Before further analyzes, we checked whether the DL of any participant in any condition violated the 3 sigma rule (over all participants). None of the participants did so.

An ANOVA with factors of standard duration and validity was performed on RT and percent correct for the one half of participants who had performed the letter discrimination task. Figure 2 depicts mean RT as a function of standard duration and validity. Mean RT was shorter on validly cued trials $(667 \mathrm{~ms})$ than on invalidly cued ones $(700 \mathrm{~ms}), F(1,11)=$ $6.83, p=.024$. Neither the main effect of standard duration, $F(1,11)=0.19, p=.674$, nor the interaction of both factors, $F(1,11)=1.36, p=.269$, approached statistical significance. A similar ANOVA on percent correct did not reveal any significant effects (all $p \mathrm{~s}>.2$ ), so that there was no speedaccuracy trade-off. The decrease of RT for validly cued trials reflects the automatic attraction of attention by an exogenous luminance cue and shows that our cuing manipulation was successful. As has been shown before (Müller \& Rabbitt, 1989), a luminance cue attracts attention automatically, and this increase in attention then facilitates processing of the comparison stimulus and decreases RT.

Separate ANOVAs with factors of letter discrimination, standard duration, and validity were performed on $\mathrm{CE}$ and DL. Figure 3 depicts mean $\mathrm{CE}$ and $\mathrm{DL}$ as a function of

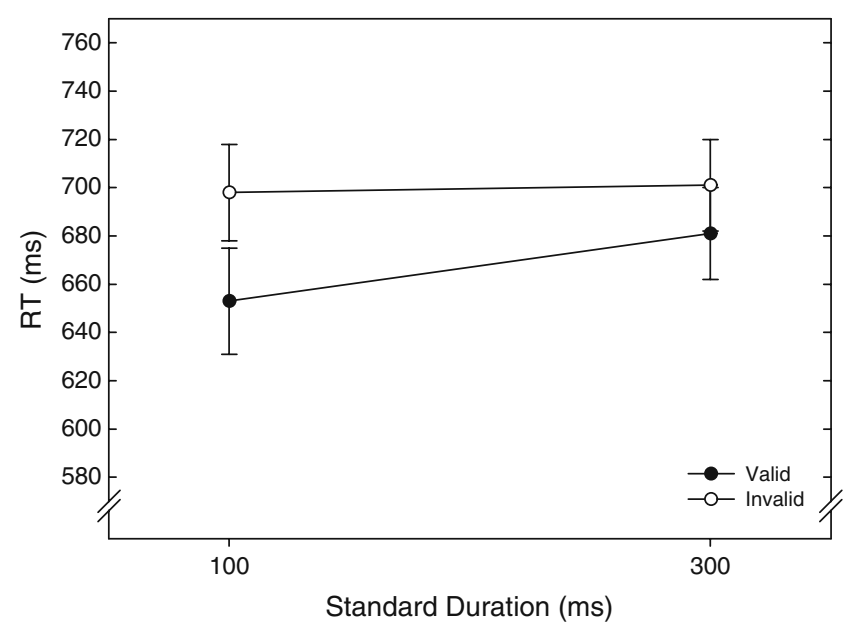

Fig. 2 Results of Experiment 1: Mean RT (in milliseconds) as a function of standard duration and validity. The error bars indicate the standard errors of the means for a within-subjects design (see Cousineau, 2005) 
letter discrimination, standard duration, and validity. The overall $\mathrm{CE}$ was positive $(44 \mathrm{~ms})$, which indicates that, in general, participants judged the comparison to be shorter than the standard duration. This finding reflects the usual underestimation of the second of two short intervals that has often been observed in experiments on time perception (e.g., Allan, 1977; Hellström \& Rammsayer, 2004).

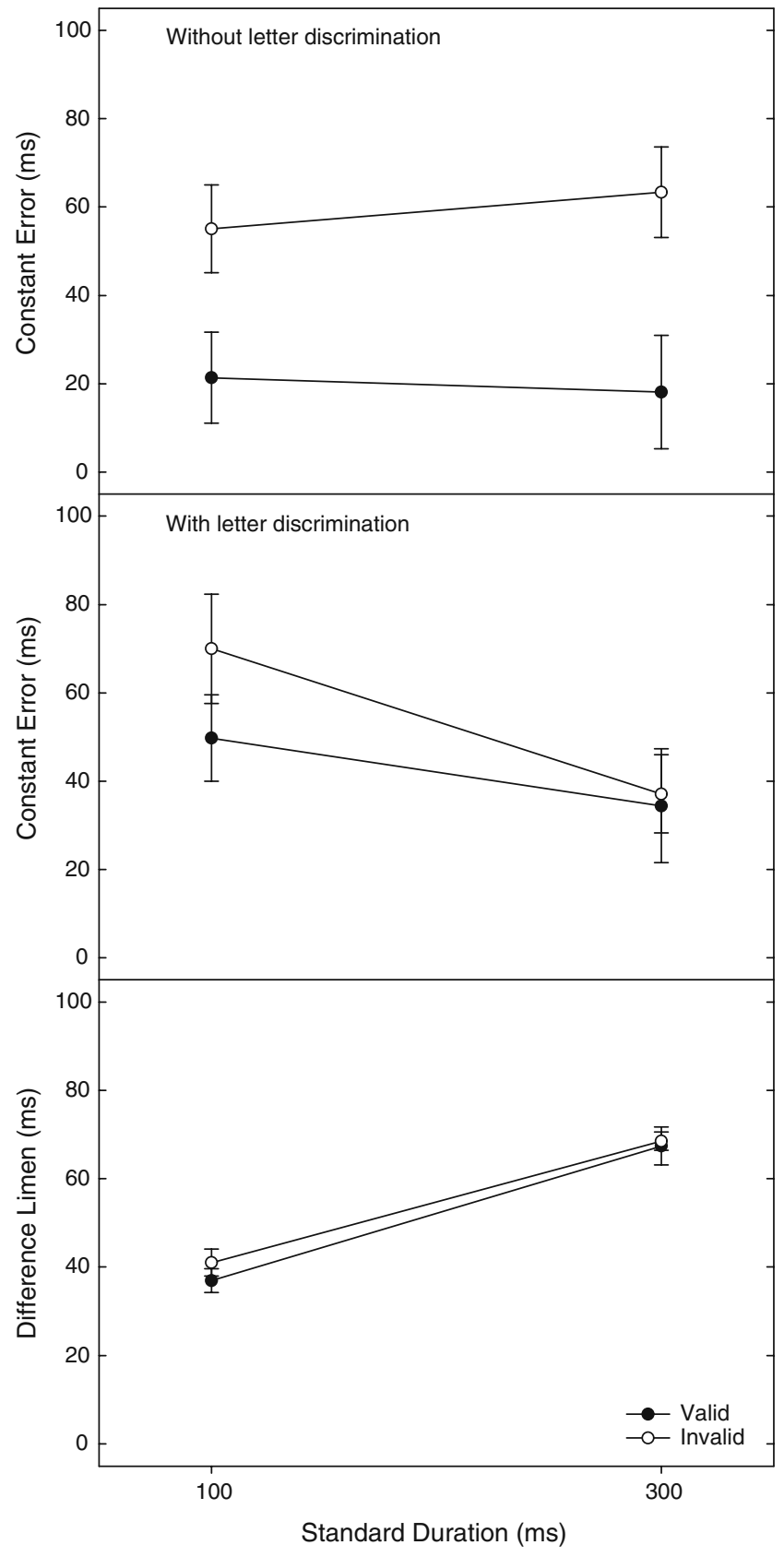

Fig. 3 Results of Experiment 1: Mean constant error and difference limen as a function of standard duration and validity. The upper panel depicts the constant error for the condition without the letter discrimination task. The middle panel depicts the constant error for the condition with the letter discrimination task. The lower panel depicts the difference limen. The error bars indicate the standard errors of the means for a within-subjects design (see Cousineau, 2005)
Theoretically more crucially, CE was smaller on validly cued trials $(31 \mathrm{~ms})$ than on invalidly cued ones $(56 \mathrm{~ms})$, $F(1,22)=15.24, p<.001$. Consequently, participants judged validly cued stimuli to be longer than invalidly cued ones. This effect, however, was modulated by the letter discrimination task, $F(1,22)=4.62, p=.043$. The difference between validly and invalidly cued trials was much less pronounced when participants had to perform the speeded letter discrimination task (mean attention effect of $11 \mathrm{~ms}$ with letter discrimination vs. $39 \mathrm{~ms}$ without letter discrimination). As Figure 3 shows, this validity effect decreased for both standard durations; for the 300-ms standard duration, it was virtually nonexistent. This difference in result patterns - that is, the three-way interaction - was, however, only marginally significant, $F(1,22)=$ $3.22, p=.087$. None of the other main effects or interactions approached statistical significance, all $p \mathrm{~s}>.3$.

In order to clarify the observed interaction between letter discrimination and validity, we submitted the $\mathrm{CE}$ data to separate ANOVAs for both groups. In the group without letter discrimination, participants judged validly cued stimuli to be longer than invalidly cued ones, $F(1,11)=18.93, p=.001$ (all other $p \mathrm{~s}>.3$ ). In the group with letter discrimination, this effect was not significant, $F(1,11)=1.49, p=.248$, nor was the interaction between validity and standard duration, $F(1,11)=2.08, p=.177$. This means that in our experiment, spatial attention prolonged perceived duration, but only when duration judgment was the only task. Then it was, however, comparable for the short and for the long standard duration. This is consistent with Experiment 5 in Mattes and Ulrich (1998), and also the size of the effect is comparable to that in this experiment. When letter discrimination had to be performed first, the attention effect could not be observed. Crucially, these results do not replicate the findings of Chen and O'Neill (2001). Whereas these authors found the attention effect only when the processing load was highthat is, when the participants had to perform a letter discrimination task - we found no attention effect on perceived duration under such circumstances.

An ANOVA on DL produced only a significant effect of standard duration, $F(1,22)=38.27, p<.001$ (all other $p$ s > .4). Consistent with Weber's law, DL was smaller for the short standard duration than for the long one (39 vs. $68 \mathrm{~ms}$ ). The corresponding Weber fractions are 0.39 and 0.23 and, therefore, are comparable with the Weber fractions on visual time perception found so far (Enns et al., 1999; Mattes \& Ulrich, 1998).

\section{Experiment 2}

Experiment 1 could not verify the account by Chen and O'Neill (2001) that spatial attention exerts its prolonging 
effect on perceived duration only with a concurrent nontemporal task. The reason for the reversed attention effect on perceived duration reported by Chen and O'Neill is therefore still unclear. Thus, in Experiment 2 , we investigated the setup of the cuing display as a potential source for the reversed effect. Chen and O'Neill also investigated whether object-based attention might influence perceived duration. Therefore, they employed the two-rectangle paradigm of object-based attention (Egly, Driver, \& Rafal, 1994; Marrara \& Moore, 2003). Even though Chen and O'Neill did not find any object-based attention effects on perceived duration, the special setup of this display may have influenced the quality of the attention effect.

In fact, perceived duration has been shown to be amenable to subtle physical differences, such as size (Thomas \& Cantor, 1975), length (Casasanto \& Boroditsky, 2008), intensity (Brigner, 1986), and number of stimuli (Xuan et al. 2007). Thus, it seems reasonable to consider the context - that is, the cuing display - when investigating how the attention effect on perceived duration might have reversed. Experiment 2 therefore compared the attention effect for a standard cuing display with two position frames (e.g., Hein, Rolke, \& Ulrich, 2006; Müller \& Rabbitt, 1989) with that for a display that comprised only one rectangular frame and, thereby, resembled the rectangles in the tworectangle method employed by Chen and O'Neill (2001). Experiment 2 employed no condition with letter discrimination, but all the participants were confronted with square and rectangular position frames, and duration judgment was the sole task they had to perform.

\section{Method}

\section{Participants}

Eighteen female and 6 male students participated in this experiment. Their ages ranged from 19 to 32 years $(M=22.5, S D=3.2)$. All but 4 participants were righthanded, and all had normal or corrected-to-normal vision. As before, they were told that the experiment was about time perception but were left naïve as to the hypotheses of the experiment. Each student took part in one experimental session consisting of two parts and received $€ 11$ or course credit.

\section{Stimuli and apparatus}

The stimuli and apparatus were identical to those in Experiment 1, except for the following changes. Apart from the cuing display from Experiment 1 with two square position frames, we also employed a display that contained only one rectangular position frame. This frame had a size of $2.88^{\circ} \times 11.45^{\circ}$ and covered the same space on the display as the square frames. This time, the comparison stimulus was identical to the standard stimulus; that is, both were gray disks.

\section{Procedure}

Figure 4 shows the display setups in Experiment 2. The procedure was identical to that in Experiment 1, except for the following changes. The cue consisted of a brightening of the three outer edges of the position frame(s) on the left or the right side.

Unlike in Experiment 1, no response prompt was used, and the trial ended with the position frame(s), which stayed on screen until the participant had judged the comparison stimulus as being either "shorter" or "longer" than the standard stimulus. Each experimental part comprised 2 (valid vs. invalid) $\times 2$ (squares vs. rectangle) $\times 2$ (cue left vs cue right) $\times 2$ (upper run vs. lower run) $\times 25=400$ trials. It started with 32 practice trials, and then participants completed 10 experimental blocks of 40 trials.

This experiment factorially combined the three withinsubjects factors of standard duration (100 vs. $300 \mathrm{~ms})$, validity (valid vs. invalid), and display (squares vs. rectangle). CE and DL were the dependent variables.

\section{Results and discussion}

Before further analyses, we checked whether the DL of any participant in any condition violated the 3 sigma rule. This was true for 3 participants, and they were replaced by 3 other participants. Separate ANOVAs with factors of standard duration, validity, and display were performed on $\mathrm{CE}$ and DL. Figure 5 depicts mean $\mathrm{CE}$ as a function of standard duration, validity, and display (upper panel), as well as DL as a function of standard duration and validity (lower panel). Mean CE was smaller $(22 \mathrm{~ms})$ on validly cued trials than on invalidly cued trials $(40 \mathrm{~ms}), F(1,23)=$ $10.12, p=.004$. Participants judged the duration of validly cued stimuli to be longer than the duration of invalidly cued ones, which replicated the results of Experiment 1; that is, spatial attention prolonged perceived duration. Theoretically most important, there was no interaction of validity and display, $F(1,23)=0.13, p=.726$. Consequently, the attention effect was independent of the display in which spatial attention was manipulated. Although the attention effect was unaffected by display, CE was smaller in the squared condition $(28 \mathrm{~ms})$ than in the rectangle condition (34 ms), $F(1,23)=7.34, p=.012$. This means that when the comparison stimuli were presented in squared position frames, participants judged them to be longer (than the standard) than when they were presented in a rectangular position frame. This main effect, however, is difficult to 
Fig. 4 The trial sequence in Experiment 2: On the upper lines, the sequence for the squared condition, and on the lower lines, the sequence for the rectangular condition (both sequences show a validly cued trial)

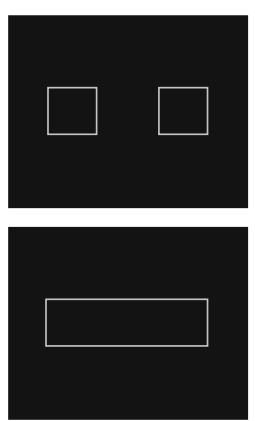

$1,000 \mathrm{~ms}$
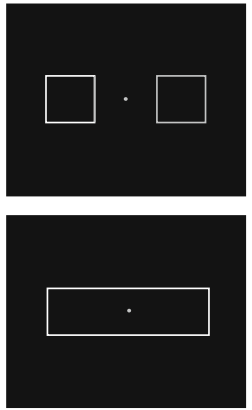

Cue: $53 \mathrm{~ms}$
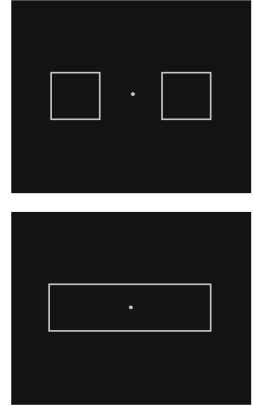

$1,000 \mathrm{~ms}$
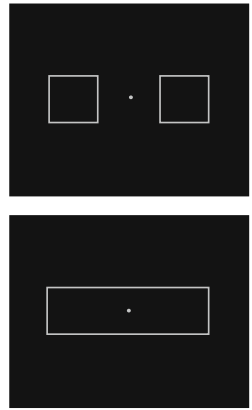

$53 \mathrm{~ms}$
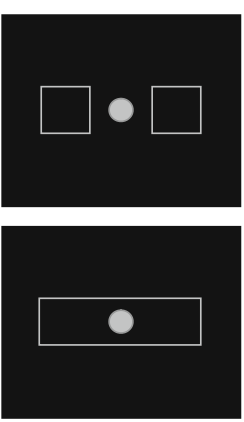

Standard duration
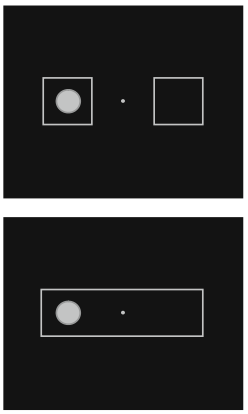

Comparison duration
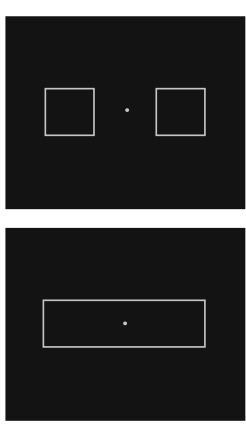

$1,000 \mathrm{~ms}$
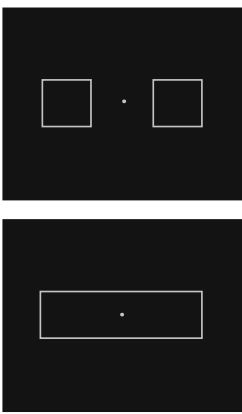

Until response interpret, because the standard stimulus also appeared in different contexts in both stimulus displays. Thus, these contexts may have affected not only the perceived duration of the comparison, but also the perceived duration of the standard. Most crucially, however, neither display produced a reversed attention effect. None of the other main effects or interactions reached statistical significance (all $p \mathrm{~s}>.5$ ).

An ANOVA on DL produced only a significant effect of standard duration, $F(1,23)=57.20, p<.001$ (all other $p \mathrm{~s}>.3$ ). Consistent with Weber's law, DL was smaller for the short standard duration than for the long one ( $26 \mathrm{vs} .62 \mathrm{~ms}$ ). The corresponding Weber fractions are 0.26 and 0.21 .

\section{Experiment 3}

Neither Experiment 1 nor Experiment 2 could clarify why Chen and O'Neill (2001) found a reversed attention effect on perceived duration, contradicting the usual effect found by Enns et al. (1999), Mattes and Ulrich (1998), and Yeshurun and Marom (2008). The results of Experiment 2 suggest that the cuing display can at least somehow influence perceived duration. In Experiment 3, we therefore used a cuing display that resembled the one used by Chen and O'Neill even more closely. Specifically, we also employed a two-rectangle paradigm that is usually used to assess object-based attention effects on RT (Egly et al.,
1994; Marrara \& Moore, 2003). We tried to resemble not only the display by Chen and O'Neill, but also their specific experimental conditions.

In this paradigm, two rectangles are located to the left and to the right of (or below and above) the fixation point. One of the four ends of the rectangles is cued with a luminance cue. A target stimulus then appears at one of these four possible ends. In line with the spatial attention effect, RT is usually found to be shorter for the validly cued stimulus than for the invalidly cued ones. To sum up, in the present experiment, we further investigated whether the reversed attention effect can be attributed to the special cuing display associated with this paradigm.

Method

\section{Participants}

Nineteen female and 5 male students participated in this experiment. Their ages ranged from 19 to 50 years $(M=24.0, S D=6.6)$. All but 2 participants were righthanded, and all had normal or corrected-to-normal vision. They were told that the experiment was about time perception but were left naïve as to the hypotheses of the experiment. Each student took part in one experimental session consisting of two parts and received $€ 9$ or course credit. 


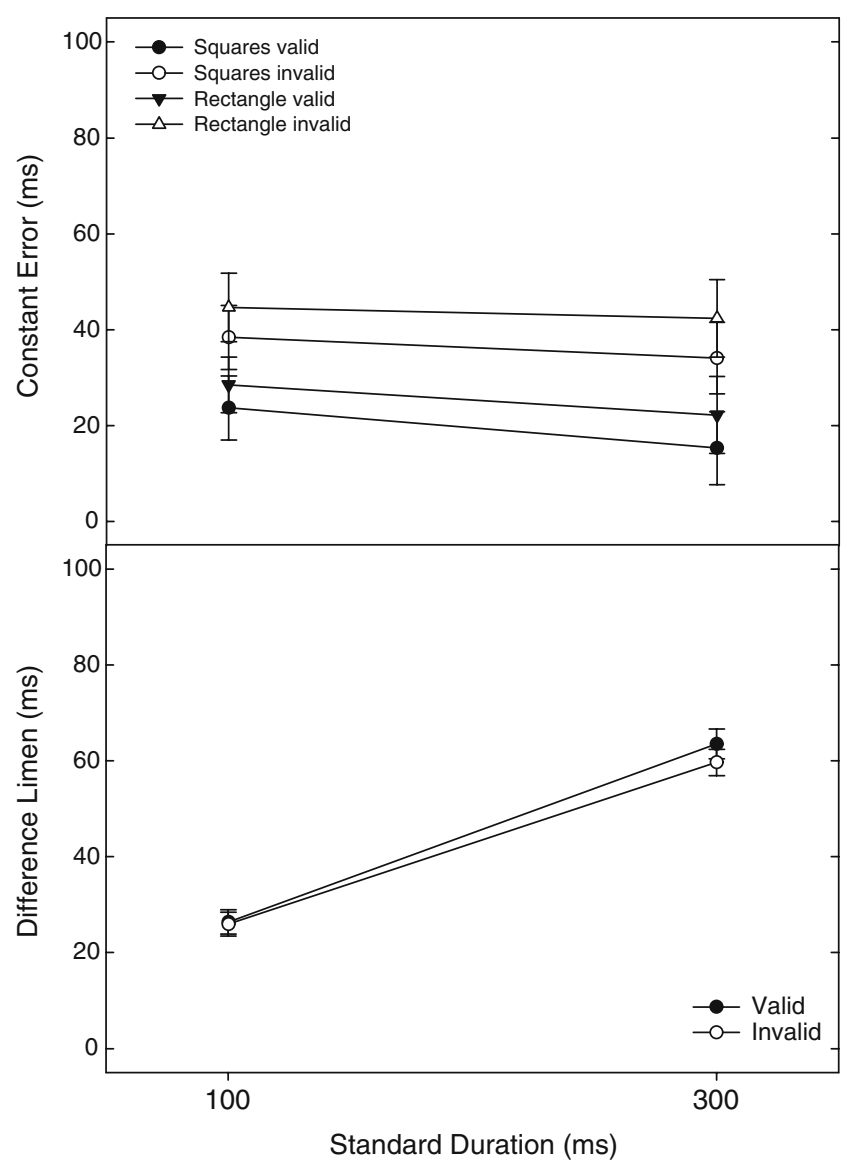

Fig. 5 Results of Experiment 2: Mean constant error (upper panel) and mean difference limen (lower panel) as a function of standard duration, validity, and display (only constant error). The error bars indicate the standard errors of the means for a within-subjects design (see Cousineau, 2005)

\section{Stimuli and apparatus}

The stimuli and apparatus were identical to those in Experiment 2, except for the following changes. Two rectangular frames appeared now on screen, both of which had the same size as the rectangular position frame in Experiment 2. Those two frames appeared either to the left and right of the fixation point (vertical orientation) or above and below the fixation point (horizontal orientation). The eccentricity was $4.29^{\circ}$ in both cases. The comparison stimuli appeared at one of the four ends of the two rectangles.

\section{Procedure}

Figure 6 shows an example trial of Experiment 3. The procedure was identical to that in Experiment 2, except for the following changes. The cue was a brightening of one of the four ends of the two rectangles. The comparison stimulus could then appear at the cued location (valid condition), at the other end of the same rectangle (invalid but same-object condition), at the adjacent location in the other rectangle (invalid and different-object condition), or at the diagonally opposing end in the other rectangle (far condition). All validity conditions had, therefore, an equal probability of $25 \%$. Again, each participant completed two experimental parts, one with a standard duration of $100 \mathrm{~ms}$ and the other one with a standard duration of $300 \mathrm{~ms}$. Each experimental part consisted of 4 (valid vs. invalid same, invalid different vs. far) $\times 4$ (cue upper left vs. cue lower left, cue upper right vs. cue lower right) $\times 2$ (orientation: horizontal vs. vertical) $\times 2$ (upper run vs. lower run) $\times 6=384$ trials. It started with 32 practice trials (16 per horizontal and vertical orientations of the two rectangles each), and then participants completed 12 experimental blocks of 32 trials. The orientation of the rectangles was varied blockwise, with one half of the participants starting with the horizontal orientation and the other half starting with the vertical orientation.

This experiment factorially combined the two withinsubjects factors of validity (valid, invalid same, invalid different, vs. far) and standard duration (100 vs. $300 \mathrm{~ms}$ ). $\mathrm{CE}$ and DL were the dependent variables.
Fig. 6 The (vertical) trial sequence in Experiment 3: Invalid different trial

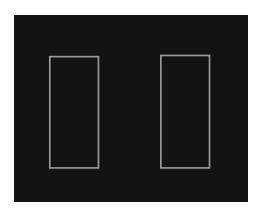

$1,000 \mathrm{~ms}$

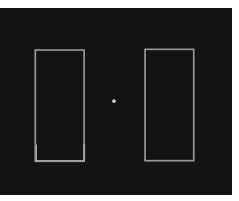

Cue: $53 \mathrm{~ms}$

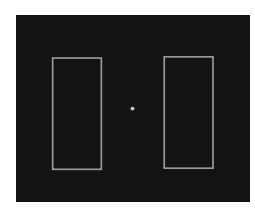

$1,000 \mathrm{~ms}$

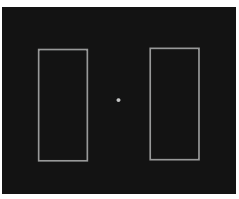

$53 \mathrm{~ms}$

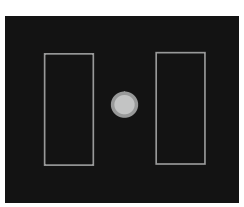

Standard duration

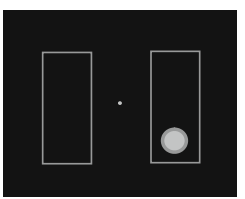

Comparison duration

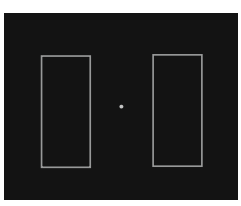

$1,000 \mathrm{~ms}$

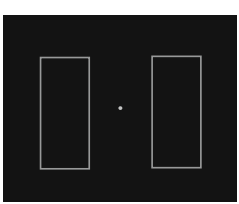

Until response 


\section{Results and discussion}

Before further analyses, we checked whether the DL of any participant in any condition violated the 3 sigma rule. This was true for 2 participants, and they were replaced. Separate ANOVAs with factors of validity and standard duration were performed on CE and DL. Whenever appropriate, $p$ values were adjusted for violations of the sphericity assumption, using the Greenhouse-Geisser correction. Figure 7 depicts mean $\mathrm{CE}$ and $\mathrm{DL}$ as a function of validity and standard duration. For CE, we observed a main effect of validity, $F(3,69)=3.46, p=.046 . \mathrm{CE}$ in the valid condition $(29 \mathrm{~ms})$ was smaller than $\mathrm{CE}$ in the other conditions taken together, as a Helmert contrast showed, $F(1,23)=4.79, p=.039$. The main effect of standard duration, as well as the interaction, did not approach statistical significance (all $p \mathrm{~s}>.4$ ).

An ANOVA on DL produced only a significant effect of standard duration, $F(1,22)=76.06, p<.001$ (all other $p \mathrm{~s}>.5)$. Consistent with Weber's law, DL was smaller for the short standard duration than for the long one ( 39 vs. $70 \mathrm{~ms})$. The corresponding Weber fractions are 0.39 and 0.24 .

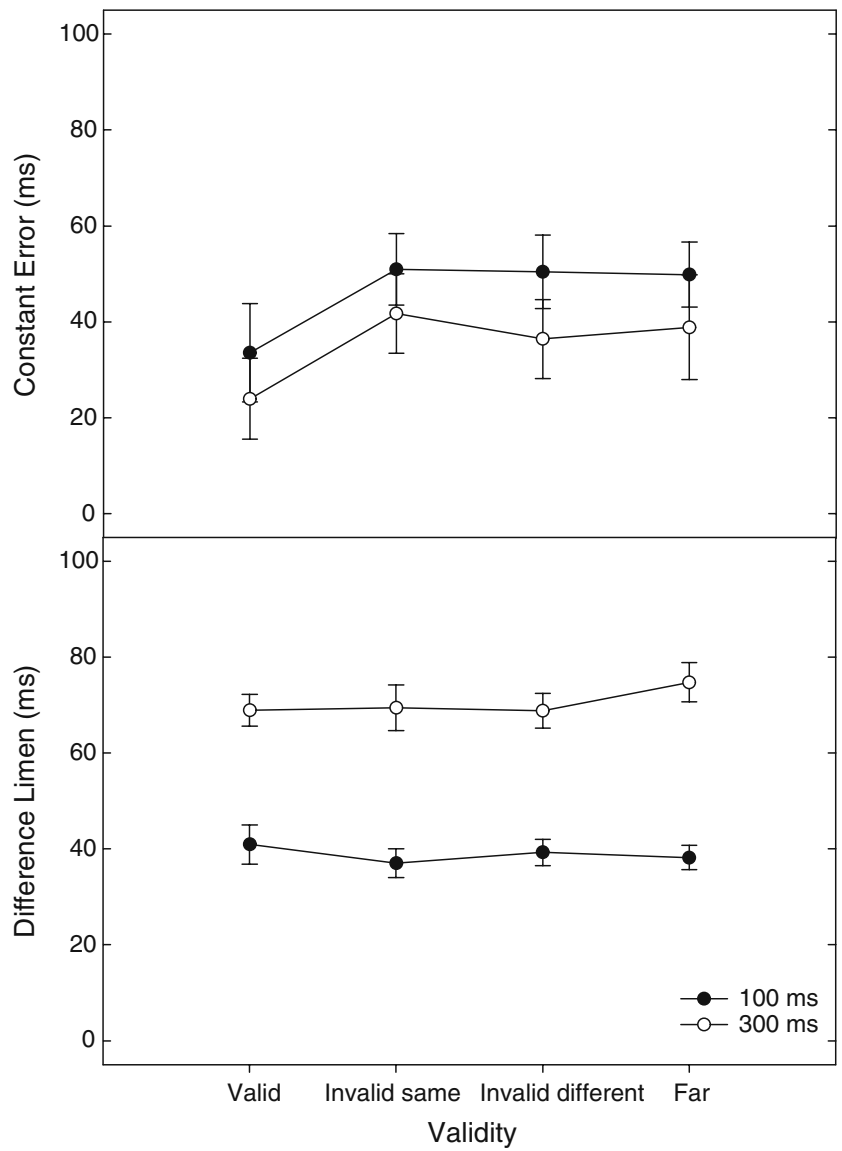

Fig. 7 Results of Experiment 3: Mean constant error (upper panel) and mean difference limen (lower panel) as a function of validity and standard duration. The error bars indicate the standard errors of the means for a within-subjects design (see Cousineau, 2005)
In sum, then, as in Experiments 1 and 2, the results of Experiment 3 confirm that orienting attention to a certain location in the visual field prolongs the perceived duration of visual stimuli presented there. This again contradicts the reversed attention effect found by Chen and O’Neill (2001).

\section{Experiment 4}

So far, the present experiments excluded processing load, cuing display, and the two-rectangle display in particular as possible sources for the reversed attention effect observed by Chen and O'Neill (2001). A further potential source could be the method with which perceived duration was measured. On the one hand, Yeshurun and Marom (2008), as well as the present experiments, employed a pair comparison method, in which participants judged whether one stimulus appeared to them shorter or longer than another one. On the other hand, Chen and O'Neill used a temporal categorization task. In this task, they presented the participants only one temporal interval per trial and asked them to classify this interval as "short", "medium", or "long". In Experiment 4, we assessed whether the reversed attention effects could be attributed to this difference in methodology, because several psychophysical studies have suggested that the results of such studies may depend on the exact judgment task (e.g., Allan, 1979; Schneider \& Komlos, 2008; Zakay, 1993).

Method

\section{Participants}

Twenty-three female and 1 male student participated in this experiment. Their ages ranged from 18 to 29 years $(M=$ $21.1, S D=2.9$ ). All but 1 participant were right-handed, and all had normal or corrected-to-normal vision. They were told that the experiment was about time perception but were left naïve as to the hypotheses of the experiment. Each student took part in one experimental session and received $€ 7$ or course credit.

\section{Stimuli, apparatus, and procedure}

The present experiment resembled closely the ones reported by Chen and O'Neill (2001). The stimuli, apparatus, and procedure were identical to those in Experiment 3, with the following exceptions. First, no standard stimulus was employed. Specifically, a trial started with the position frames $(1,000 \mathrm{~ms})$ and continued with the position frames together with the fixation dot $(1,000 \mathrm{~ms})$, and then the cue was presented. Second, the duration of the interval to be 
judged could only have one of three possible durations (60, 107 , or $167 \mathrm{~ms}$ ). Third, after the presentation of the target stimulus, participants had to classify it as "short", "medium", or "long". For this categorization, they used the ",", "..", and "--" keys, which were labeled as "1", "2", and " 3 ", respectively. This categorization was followed by an ITI of $1,000 \mathrm{~ms}$, and then the next trial started. Finally, at the beginning of the experimental session, each participant was familiarized with the three target durations. Four example trials of each duration, labeled as "short", "medium", and "long", were shown to the participants. After these 12 trials, the participants were asked whether they had noticed any difference between the three durations. If they had not, the example trials were presented again (note that none of our participants needed more than two repetitions of the 12 example trials).

The experimental session consisted of 4 (valid vs. invalid same, invalid different vs. far) $\times 4$ (cue upper left vs. cue lower left, cue upper right vs. cue lower right) $\times 2$ (horizontal vs. vertical ) $\times 3$ (duration: short vs. medium vs. long) $\times 6=576$ trials. It started with 48 practice trials ( 24 for each of the two orientations of the two rectangles), and then participants completed 12 experimental blocks of 48 trials. The orientation of the rectangles was varied blockwise, with one half of the participants starting with the horizontal orientation and the other half starting with the vertical orientation. Thus, this experiment factorially combined the two within-subjects factors of validity (valid vs. invalid same, invalid different vs. far) and duration (short vs. medium vs. long). Categorized duration was the dependent variable.

\section{Results and discussion}

An ANOVA with factors of validity and duration was performed on categorized duration. Figure 8 shows catego-

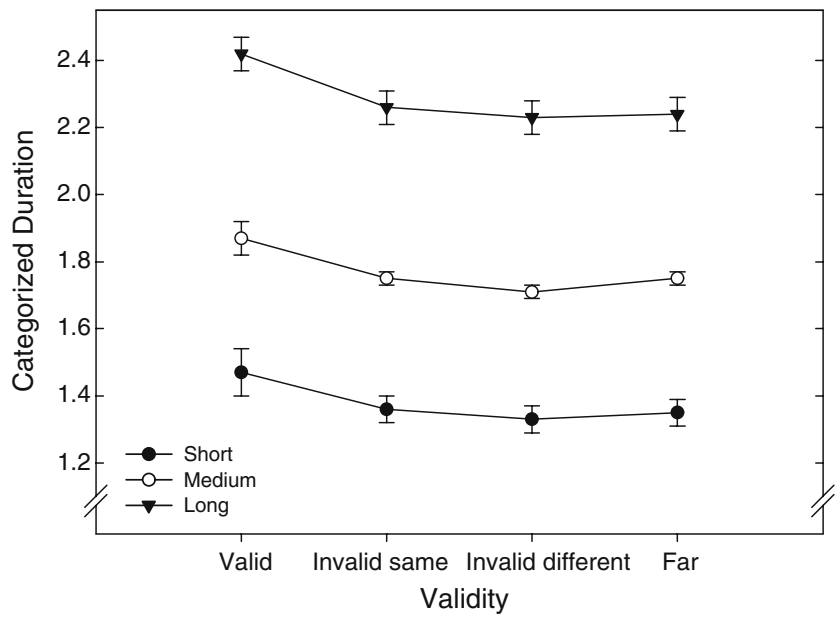

Fig. 8 Results of Experiment 4: Categorized duration as a function of validity and duration. The error bars indicate the standard errors of the means for a within-subjects design (see Cousineau, 2005) rized duration as a function of validity and duration. As one would expect, we observed a main effect of duration, $F(2,46)=135.81, p<.001$, showing that participants were clearly able to discriminate between the three durations. Categorized duration increased with duration (categorized durations for short, medium, and long: 1.38, 1.77, and $2.29)$, which was also shown by a significant linear trend, $F(1,69)=271.60, p<.001$.

Theoretically most important, there was also a main effect of validity, $F(3,69)=5.51, p=.021$, but the interaction between duration and validity was not significant, $F(6,138)=0.64, p=.628$. Participants rated the target duration as longer (1.92) in the valid condition than in the other invalid conditions taken together, as a Helmert contrast showed, $F(1,23)=5.90, p=.023$. Consequently, the same objective duration was judged longer when it was presented at an attended location in visual space. Therefore, even with the same display setup and the same method as that used by Chen and O'Neill (2001), the present results are clearly at variance with their reversed attention effect.

\section{Experiment 5}

After the exclusion of the measurement method, a last potential source for the reversed attention effect is the type of cue. Yeshurun and Marom (2008) employed a bar cue, and the cue in the present experiments was a white luminance cue - that is, a brief brightening of the frame contours. In contrast, the cue in Chen and O'Neill (2001) was a filled square that covered the same area as the target stimulus. Research has shown that the type of cue can be an important determinant for the observation of cuing effects on RT (McAuliffe \& Pratt, 2005; Pratt, Hillis, \& Gold, 2001). Pratt et al., for example, employed a detection task and varied whether the cue and target stimulus spatially overlapped or not. Crucially, shorter RTs at the validly cued target position were observed only when the cue and target did not spatially overlap. Even though the RT effect did not reverse, as was the case for the effect on perceived duration in Chen and O'Neill, it seems worthwhile to take the type of cue into account. Hence, we re-ran Experiment 4, but with a filled gray cue instead of our white luminance cue.

Method

\section{Participants}

Twenty-five female and 9 male students participated in this experiment. Their ages ranged from 19 to 38 years $(M=23.4, S D=4.3)$. All but 2 participants were righthanded, and all had normal or corrected-to-normal vision. They were told that the experiment was about time 
perception but were left naïve as to the hypotheses of the experiment. Each student took part in one experimental session and received $€ 7$ or course credit.

\section{Stimuli, apparatus, and procedure}

The stimuli, apparatus, procedure, and design were identical to those in Experiment 4, except for the cue. Instead of a brightening of the three outer edges of the position frames, the whole squared end of a rectangular frame $\left(2.88^{\circ} \times 2.88^{\circ}\right)$ was briefly illuminated in the same gray shade that all the other stimuli were presented in. In this experiment, no participant needed more than three repetitions of the 12 example trials.

Results and discussion

An ANOVA with factors of validity and duration was performed on categorized duration. Whenever appropriate, $p$ values were adjusted for violations of the sphericity assumption, using the Greenhouse-Geisser correction. Figure 9 depicts categorized duration as a function of validity and duration. As one would expect and in line with Experiment 4, we observed a main effect of duration, $F(2,66)=186.95, p<.001$. Hence, participants were again able to discriminate between the three target durations. Crucially, however, there was no significant effect of validity, $F(3,99)=0.61, p=.454$, and also no significant interaction, $F(6,198)=2.14, p=.093$. That is, in line with research on RT (McAuliffe \& Pratt, 2005; Pratt et al., 2001), there was no effect on perceived duration when the cue and target stimulus spatially overlapped. Note, however, that a closer inspection of the data showed that this insignificant effect of validity was most likely not due to a lack of difference between the valid and invalid conditions. Rather,

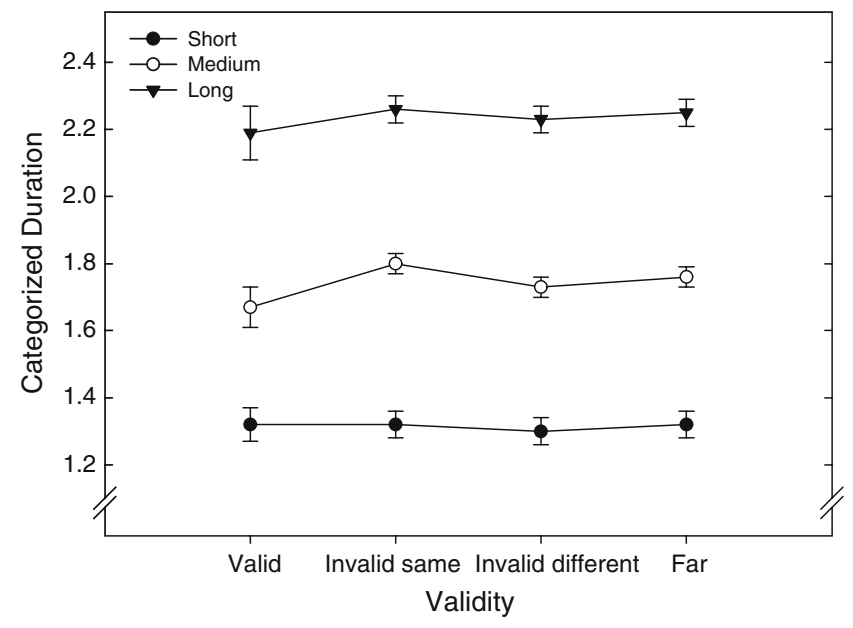

Fig. 9 Results of Experiment 5: Categorized duration as a function of validity and duration. The error bars indicate the standard errors of the means for a within-subjects design (see Cousineau, 2005) some participants showed increased ratings for the valid condition, as compared with the invalid ones, and some participants showed the opposite pattern. When we compared the mean for the valid condition with the mean for all the invalid conditions together, 16 participants had a higher rating in the valid condition than in the invalid ones, and for the remaining 18 participants, this pattern was reversed. ${ }^{4}$

To sum up, Experiment 5, too, cannot definitely dissolve the reversed attention effect observed by Chen and O'Neill (2001). However, it provides suggestive evidence that the type of cue might have some influence on the attention effect on perceived duration.

\section{Experiment 6}

The previous experiments suggest that exogenous attention prolongs, rather than shortens, perceived duration. Nevertheless, it is possible to attribute this effect to a decision bias, rather than to a genuine attention effect on perceived duration. Specifically, Schneider and Komlos (2008) argued that cuing paradigms in combination with comparative judgments, as used in the previous experiments, can be prone to criterion effects. Schneider and Komlos argued that attention, as directed by a cue, might not necessarily influence perceived duration but could also change the response pattern by influencing decision mechanisms. In short, attention might prioritize the corresponding target and increase participants' preference for a "longer" response.

Schneider and Komlos (2008) demonstrated, within the framework of a plausible mathematical model, that an equality judgment, rather than a comparison judgment, can deconfound such a criterion and genuine attention effects. Therefore, in Experiment 6, we employed an equality

\footnotetext{
$\overline{{ }^{4} \text { Experiment } 5}$ suggested that the effect of exogenous cuing on perceived duration varies between participants. In order to reveal whether a similar pattern was present in the preceding experiments, we rechecked the data for individual differences. For each experiment, we compared the measure of perceived duration (i.e., $\mathrm{CE}$ or categorized duration) in the valid condition with that in the invalid condition(s). More specifically, this comparison was performed for each factorial combination within each experiment (excluding Experiments 4 and 5 and the experiment reported in Note 3, for which the mean for the valid condition was compared with the mean for all the invalid conditions). In Experiment 1, there was a total of 48 possible comparisons, and the majority of these comparisons (77.1\%) showed the expected attention effect. The corresponding number of comparisons and percentages in Experiments 2, 3, 4, and 6 were 96/70.8\%, $48 / 60.4 \%, 24 / 70.8 \%$, and $12 / 83.3 \%$, respectively. In the experiment reported in Note 3 , however, the majority of comparisons $(73.3 \%$ of 30 comparisons) showed the reversed attention effect. Thus, there were individual differences in each of the experiments; however, the percentage of participants that showed the usual attention effect clearly prevailed.
} 
judgment analogous to that in the study of these authors. If the prolonging effect in the previous experiments was a genuine attention effect, one would expect it to persist also when participants have to judge the equality of a standard and a comparison stimulus. Consequently, this experiment considerably extended the scope of the previous experiments, because it examined not only the direction of the attention effect, but also its nature.

Method

\section{Participants}

Eight female and 4 male students participated in this experiment. Their ages ranged from 21 to 27 years $(M=23.8, S D=2.5)$. All but 3 participants were righthanded, and all had normal or corrected-to-normal vision. They were told that the experiment was about time perception but were left naïve as to the hypotheses of the experiment. Each student took part in one experimental session and received $€ 7$ or course credit.

\section{Stimuli, apparatus, and procedure}

The stimuli, apparatus, and trial structure were similar to those in Experiments 1 and 2, with the following specific properties. Two square position frames, as in Experiment 1, were employed, and the cue was likewise a brief brightening of the contours of one of the frames. The standard and comparison stimuli were gray disks, as in Experiment 2, and we employed only one standard duration of $300 \mathrm{~ms}$. The comparison durations were determined according to a method of constant stimuli. Specifically, the comparison stimulus could have a duration of 113, 160, 207, 253, 300, $347,393,440$, or $487 \mathrm{~ms}$; that is, its durations varied symmetrically around the standard duration.

At the end of one trial, the position frames stayed on screen until the participant had judged whether the standard stimulus and the comparison stimulus were either "equal" in duration or "unequal." Participants indicated their responses by pressing either the left or the right shift key, respectively.

This experiment comprised 2 (valid vs. invalid) $\times 9$ (comparison durations) $\times 2$ (cue left vs. cue right) $\times 10=$ 360 trials. It started with 36 practice trials, and then participants completed 10 experimental blocks of 36 trials. Taken together, we collected 20 judgments for each factorial combination of validity and comparison duration. Thus, the experimental design involved the two withinsubjects factors of comparison duration (113 vs. 160 vs. 207 vs. 253 vs. 300 vs. 347 vs. 393 vs. 440 vs. $487 \mathrm{~ms}$ ) and validity (valid vs. invalid). The dependent variable was the relative frequency of "equal" responses.
Results and discussion

The upper panel of Figure 10 shows the relative frequency of "equal" judgments $(f)$ as a function of comparison duration. An ANOVA with factors of comparison duration and validity was performed on $f$. As one would expect, $f$ varied with comparison duration, $F(8,88)=20.09, p<.001$, showing that participants could discriminate well between the different comparison durations. Validity also produced an effect on $f, \mathrm{~F}$ $(1,11)=10.57, p=.008$; that is, the overall relative frequency of "equal" responses was greater in the valid than in the invalid condition ( 0.57 vs. 0.41 ). Most crucially, however, the ANOVA revealed a significant interaction between validity and comparison duration, $F(8,88)=3.58$, $p=.012$. An inspection of Figure 10 showed that this could have been due to a leftward shift of the valid function, which would indicate a smaller PSE and, thus, a longer perceived duration for validly cued stimuli.

A feasible approach to assessing whether the PSEs of the valid and the invalid conditions differ from each other is the

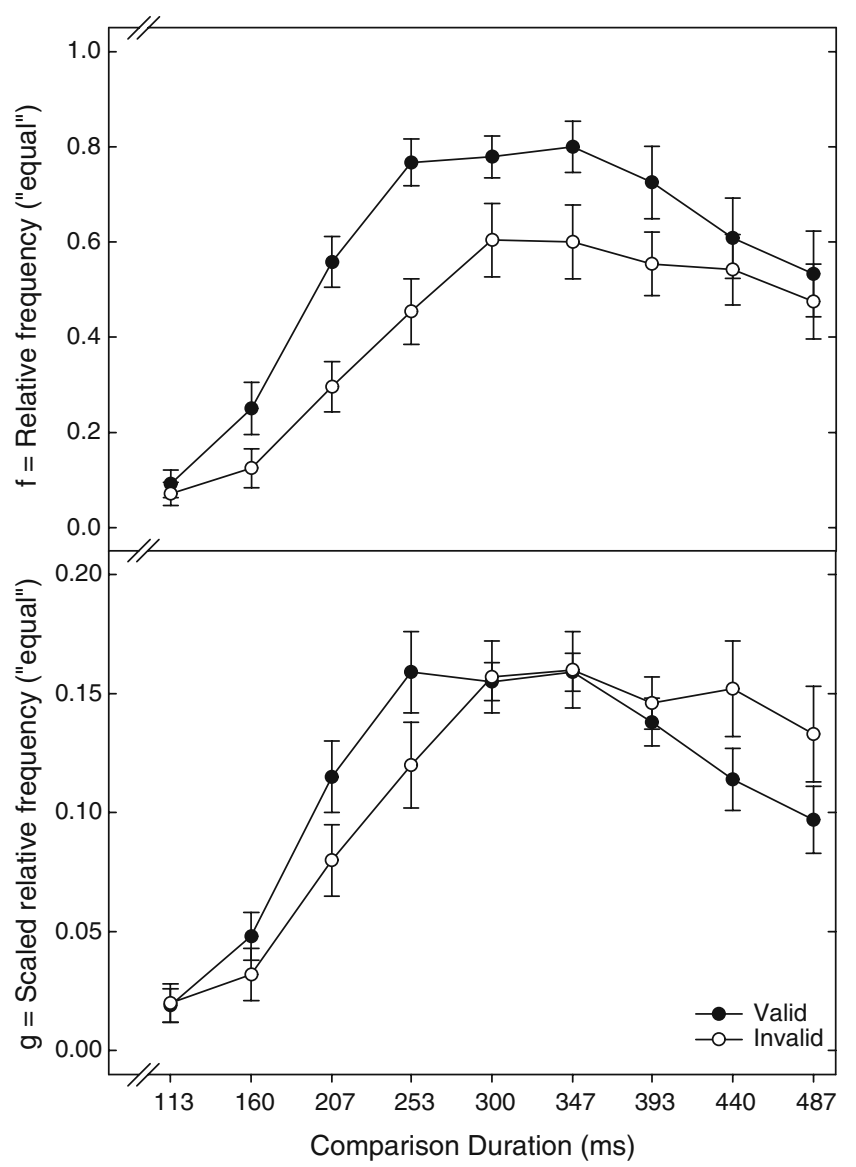

Fig. 10 Results of Experiment 6. Upper panel: Relative frequency of "equal" responses as a function of comparison duration and validity. Lower panel: Scaled relative frequency of "equal" responses as a function of comparison duration and validity. The error bars indicate the standard errors of the means for a within-subjects design (see Cousineau, 2005) 
method of waveform moments analysis (Dorfman \& Cacioppo, 1990; Ulrich, Wing, \& Rinkenauer, 1995)..$^{5}$ We used this method to compute the location of each function (i.e., the PSE) for each participant and condition. In brief, the location of a function is computed as

$P S E=\sum_{i=1}^{9} c_{i} \cdot g_{i}$

with

$g_{i}=\frac{f_{i}}{\sum_{j=1}^{9} f_{j}}$

where $c_{i}$ is the duration of the comparison stimulus and $f_{i}$ is the relative frequency of "equal" judgments associated with duration $c_{i} ; g_{i}$ denotes the scaled relative frequency of "equal" judgments, such that the scaled relative frequencies sum to 1 , just like a probability function. Therefore, Equation 2 denotes the mean of this scaled function. The mean scaled function is depicted in the lower panel of Figure 10. The mean PSEs in the valid and invalid conditions were 326 and $346 \mathrm{~ms}$, respectively, $t(11)=2.67, p=.011$ (one-tailed). This difference is consistent with the notion that validly cued stimuli are judged longer than invalidly cued ones. This result renders it unlikely that the effects observed in Experiments 1-4 were due solely to a decision bias. Rather, the result of Experiment 6 strengthens the idea that exogenous attention prolongs perceived duration.

\section{General discussion}

As was elaborated in the introduction, previous studies (Enns et al., 1999; Mattes \& Ulrich, 1998; Yeshurun \& Marom, 2008) have shown that spatial attention prolongs the perceived duration of a visual stimulus. In contrast to these studies, Chen and O'Neill (2001) reported the opposite result. The present study therefore addressed possible factors that might have contributed to the reversal of the attention effect in Chen and O'Neill's study.

In Experiment 1, we evaluated the processing load hypothesis of Chen and O'Neill (2001). The results did not support this hypothesis. In fact, we replicated the usual attention effect on perceived duration when the processing load was low. In addition, the effect was attenuated when

\footnotetext{
${ }^{5}$ In Schneider and Komlos (2008), the PSE was determined from fitting the individual data to a difference of cumulative normal distribution functions. Since the individual functions in Experiment 6 tended to be asymmetrical (such asymmetrical functions are not uncommon in the literature on perceived duration; see, e.g., Wearden, 2004), such a parametric approach was not feasible. We therefore decided to employ a nonparametrical approach to calculate the PSE of these functions.
}

the processing load was high. This is in the opposite direction from what was observed by Chen and O'Neill. That is, instead of entailing a prolonging effect of attention on perceived duration, high processing load attenuated this effect.

In Experiments 2 and 3, we examined whether the cuing display influenced the effect of spatial attention on perceived duration. In Experiment 2, one condition used a rectangular frame that was similar to the display setup in the study by Chen and O'Neill (2001). A second condition used two square frames, which have often been employed in experiments on spatial attention (Hein et al., 2006; Müller \& Rabbitt, 1989). We observed that the display setup affected perceived duration, since the stimuli presented in the square position frames were perceived to be longer than those in a rectangular frame. This effect, however, was only additive to the attention effect on perceived duration and was, therefore, also not eligible as a reason why Chen and O'Neill did not find the attention effect on perceived duration. In Experiment 3 , we investigated the attention effect on perceived duration with a two-rectangle paradigm (Chen \& O'Neill, 2001; Egly et al., 1994; Marrara \& Moore, 2003). Even under this condition, which was most similar to Chen and O'Neill's cuing display, the usual attention effect was observed; that is, validly cued stimuli were perceived to be longer than invalidly cued ones.

In Experiment 4, we focused on another important experimental aspect in the realm of perceived durationthat is, the measurement method. In contrast to the pair comparison method that we had used so far in Experiments 1-3, we now employed a temporal categorization task, as was used by Chen and O'Neill (2001). However, also with this different measurement method, we could replicate our finding from the previous experiments-that is, exogenous attention prolonged perceived duration.

Finally, in Experiment 5, we assessed whether the attention effect might change with the type of cue. In the present Experiments 1-4, as well as in the experiments by Yeshurun and Marom (2008), the cue and target did not spatially overlap. In contrast to that, and in alignment with Chen and O'Neill (2001), Experiment 5 employed a spatially overlapping cue and target. With this setup and for the first time in the present experimental series, we could not observe a prolonging effect of spatial attention on perceived duration.

Taken together, Experiments $1-5$ exclude processing load, cuing display, and measurement method as explanations for the reversed attention effect. Concerning the type of the cue, the situation is not completely clear. With a spatially overlapping cue and target, we could not detect an attention effect on perceived duration, but we also could not observe a reversed attention effect. Hence, even though we cannot provide a conclusive explanation for why Chen and 
O'Neill (2001) found a reversed attention effect on perceived duration, the present experiments nevertheless add to the body of knowledge on perceived duration. Specifically, the following conclusions can be drawn. First, we demonstrated that the prolonging effect of attention on perceived duration persists under several experimental setups and can therefore be regarded as a rather general finding. This also implies that the reversed attention effect found by Chen and O'Neill should be seen as a finding due to the specific conditions under which it was observed. In this vein, we propose, second, that the present results show that the prolonging effect of spatial attention on perceived duration might be influenced by two sets of factors that require further investigation.

The first set consists of factors known to influence perceived duration. For example, concurrent task processing (or high processing load, as in Experiment 1) is known to decrease perceived duration (e.g., Brown, 1997; Hemmes et al., 2004; Macar et al., 1994; Rammsayer \& Ulrich, 2005), since the non-temporal concurrent task detracts attention from the duration judgment task. Although this effect did not fully emerge in Experiment 1 (CE in the condition with letter discrimination was only numerically higher than that in the condition without letter discrimination, but far from significance), concurrent task processing attenuated the attention effect on perceived duration. Thomas and Weaver (1975) suggested that under concurrent task processing, more attention is allocated to a non-temporal information processor and less to a temporal information processor. When we assume that spatial attention exerts its effect via this temporal information processor, it seems likely that its effect on perceived duration is attenuated under concurrent task processing. In order to expand this finding, future research might systematically investigate the interplay between the spatial attention effect on perceived duration and other factors known to affect perceived duration (e.g., stimulus intensity, stimulus oddity).

The second set consists of factors that are known to influence the result pattern of exogenous cuing. It has been shown, for example, that when the cue and the target stimulus overlap in space, no RT decrease for validly cued stimuli is found (McAuliffe \& Pratt, 2005; Pratt et al., 2001). In the present Experiment 5 also, no effect on perceived duration was observed when the cue and the target stimulus spatially overlapped. Pratt et al. suggested that not only is spatial attention allocated to the cued position, but also sensory-based processes (e.g., more difficult cue-target discriminability, masking) become active and interfere with the attentional benefit. It is plausible that for duration judgments, such sensory processes might play an especially important role. Consequently, we did not find an attention effect on perceived duration in Experiment 5, and for similar reasons, Chen and
O'Neill (2001) may have found a reversed attention effect in their experiment. ${ }^{6}$

Another factor known to influence the pattern of results for exogenous cuing is, for example, the stimulus onset asynchrony (SOA) between the cue and the target stimulus. When the SOA increases, for example, RT becomes longer for validly cued stimuli than for invalidly cued ones; that is, the attention effect reverses (Posner \& Cohen, 1984). This inhibition of return (IOR) has been explained by an inhibition of attention's returning to a position in the visual field that has previously been attended. We suspect that the reversed attention effect that we found in the experiment mentioned in Note 3 might have been due to IOR, since the cue-target SOA was $300 \mathrm{~ms}$. In order to further examine the effect of spatial attention on perceived duration, a systematic investigation under various spatial and temporal conditions seems necessary.

As far as a theoretical account for the attention effect on perceived duration is concerned, all of the previous studies (Enns et al., 1999; Mattes \& Ulrich, 1998; Yeshurun \& Marom, 2008) have explained it in a comparable manner. The basic idea is that spatial attention prolongs the internal response that is generated by a stimulus. This account can be reconciled with the traditional pacemaker counter model of human time perception (Church, 1984; Treisman, 1963; Wearden, 1991). In this model, a pacemaker constantly emits temporal pulses that are accumulated in a counter. The more pulses are accumulated during a certain interval, the longer the perceived duration. When the internal response of a stimulus is prolonged through attention, more pulses are collected during the internal response of a stimulus, and thus the stimulus is perceived as relatively long. Therefore, the present results are consistent not only with previous studies on spatial attention, but also with common accounts on the mechanisms of time perception.

The aforementioned explanation was put to a further test in Experiment 6, whose aim was to exclude response bias as an alternative explanation. Specifically, Schneider and Komlos (2008) had suggested that cued — that is, attendedstimuli might be assigned a higher priority, giving participants a preference for "longer" responses. With a comparison judgment task, a true prolonging effect of attention on perceived duration and such a response bias cannot be distinguished. Both phenomena express themselves as a leftward shift of the psychometric function - that is, as a smaller PSE (a smaller CE). In an equality judgment task, however, a true attention effect and a response bias are represented by different characteristics of the psychometric function: a smaller PSE and a change in amplitude,

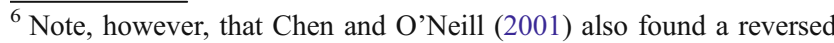
attention effect for endogenous central cues, which we cannot explain on the basis of the present results.
} 
respectively. Using such an equality judgment task in Experiment 6, we showed that the PSE shift for valid stimuli persists. Still, there was also a higher overall frequency of "equal" responses for the valid condition. Hence, apart from an effect on perceived duration, attention might also have increased participants' preference for an "equal" response.

To sum up, the present experiments suggest that the reversed attention effect on perceived duration reported by Chen and O'Neill (2001) is rather the exception than the rule. Certainly, further research is required to isolate the specific conditions that might influence the attention effect on perceived duration. Nevertheless, the present study adds to the majority of studies that have revealed that spatial attention prolongs perceived duration of visual stimuli. In addition, it extends them by excluding response bias as an alternative explanation.

Author Note This work was supported by the Deutsche Forschungsgemeinschaft (UL 116/10-1). We thank Sonja Cornelsen, Linda Idelberger, and Agnes Mercz for their assistance in data collection. We also thank three anonymous reviewers for helpful comments and suggestions on a previous version of this work. Correspondence concerning this article should be sent to Tanja Seifried, Cognitive and Biological Psychology, University of Tübingen, Friedrichstr. 21, 72072 Tübingen, Germany. E-mail: tanja.seifried@unituebingen.de

\section{References}

Allan, L. G. (1977). The time-order error in judgments of duration. Canadian Journal of Psychology, 31, 24-31. doi:10.1037/ h0081647

Allan, L. G. (1979). The perception of time. Perception \& Psychophysics, 26, 340-354.

Bashinski, H. S., \& Bacharach, V. R. (1980). Enhancement of perceptual sensitivity as the result of selectively attending to spatial locations. Perception \& Psychophysics, 28, 241-248.

Brainard, S. W. (1997). The Psychophysics Toolbox. Spatial Vision, 10, 433-436. doi:10.1163/156856897X00357

Brigner, W. L. (1986). Effect of perceived brightness on perceived time. Perceptual and Motor Skills, 63, 427-430.

Brown, S. W. (1995). Time, change, and motion: The effects of stimulus movement on temporal perception. Perception \& Psychophysics, 57, 105-116.

Brown, S. W. (1997). Attentional resources in timing: Interference effects in concurrent temporal and non-temporal working memory tasks. Perception \& Psychophysics, 59, 1118-1140.

Bush, R. R. (1963). Estimation and evaluation. In R. Luce, R. R. Bush, \& E. Galanter (Eds.), Handbook of mathematical psychology (Vol. 1, pp. 429-469). New York: Wiley.

Casasanto, D., \& Boroditsky, L. (2008). Time in the mind: Using space to think about time. Cognition, 106, 579-593. doi:10.1016/ j.cognition.2007.03.004

Chen, Z., \& O'Neill, P. (2001). Processing demand modulates the effects of spatial attention on the judged duration of a brief stimulus. Perception \& Psychophysics, 63, 1229-1238.

Church, R. M. (1984). Properties of the internal clock. Annals of the New York Academy of Sciences, 423, 566-582. doi:10.1111/ j.1749-6632.1984.tb23459.x
Cousineau, D. (2005). Confidence intervals in within-subject designs: A simpler solution to Loftus and Masson's method. Tutorials in quantitative methods for psychology, 1, 42-45.

Dorfman, D. D., \& Cacioppo, J. T. (1990). Waveform moment analysis: Topographical analysis of nonrhythmic waveforms. In J. T. Cacioppo \& L. G. Tassinary (Eds.), Principles of psychophysiology (pp. 661-707). New York: Cambridge University Press.

Egly, R., Driver, J., \& Rafal, R. D. (1994). Shifting visual attention between objects and locations: Evidence from normal and parietal lesion subjects. Journal of Experimental Psychology: General, 123, 161-177. doi:10.1037/0096-3445.123.2.161

Enns, J. T., Brehaut, J. C., \& Shore, D. I. (1999). The duration of a brief event in the mind's eye. The Journal of General Psychology, 126, 355-372. doi:10.1080/00221309909595371

Grondin, S. (1993). Duration discrimination of empty and filled intervals marked by auditory and visual signals. Perception \& Psychophysics, 54, 383-394.

Grondin, S. (2001). From physical time to the first and second moments of psychological time. Psychological Bulletin, 127, 22 44. doi: $10.1037 / 0033-2909.127 .1 .22$

Hein, E., Rolke, B., \& Ulrich, R. (2006). Visual attention and temporal discrimination: Differential effects of automatic and voluntary cueing. Visual Cognition, 13, 29-50. doi:10.1080/ 13506280500143524

Hellström, Å., \& Rammsayer, T. H. (2004). Effects of time-order, interstimulus interval, and feedback in duration discrimination of noise bursts in the 50- and 1000-ms ranges. Acta Psychologica, 116, 1-20. doi:10.1016/j.actpsy.2003.11.003

Hemmes, N. S., Brown, B. L., \& Kladopoulos, C. N. (2004). Time perception with and without a concurrent non-temporal task. Perception \& Psychophysics, 66, 328-341.

Kaernbach, C. (1991). Simple adaptive testing with the weighted updown method. Perception \& Psychophysics, 49, 227-229.

Lapid, E., Ulrich, R., \& Rammsayer, T. (2008). On estimating the difference limen in duration discrimination tasks: A comparison of the 2AFC and the reminder task. Perception \& Psychophysics, 70, 291-305. doi:10.3758/PP.70.2.291

Long, G. M., \& Beaton, R. J. (1980). The contribution of visual persistence to the perceived duration of brief targets. Perception \& Psychophysics, 28, 422-430.

Macar, F., Grondin, S., \& Casini, L. (1994). Controlled attention sharing influences time estimation. Memory \& Cognition, 22, 673-686.

Marrara, M. T., \& Moore, C. M. (2003). Object-based selection in the two-rectangles method is not an artifact of the three-sided directional cue. Perception \& Psychophysics, 65, 1103-1109.

Mattes, S., \& Ulrich, R. (1998). Directed attention prolongs the perceived duration of a brief stimulus. Perception \& Psychophysics, 60, 1305-1317.

Mayfrank, L., Kimming, H., \& Fischer, B. (1987). The role of attention in the preparation of visually guided saccadic eye movements in man. In J. K. O'Regan \& A. Levy-Schoen (Eds.), Eye movements: From physiology to cognition (pp. 37-45). Amsterdam: North-Holland.

McAuliffe, J., \& Pratt, J. (2005). The role of temporal and spatial factors in the covert orienting of visual attention tasks. Psychological Research, 69, 285-291. doi:10.1007/s00426-0040179-4

Müller, H. J., \& Rabbitt, P. M. (1989). Reflexive and voluntary orienting of visual attention: Time course of activation and resistance to interruption. Journal of Experimental Psychology: Human Perception and Performance, 15, 315-330. doi:10.1037/ 0096-1523.15.2.315

Nakayama, K., \& Mackeben, M. (1989). Sustained and transient components of focal visual attention. Vision Research, 29, 16311647. doi:10.1016/0042-6989(89)90144-2 
Pelli, D. G. (1997). The VideoToolbox software for visual psychophysics: Transforming numbers into movies. Spatial Vision, 10, 437-442. doi:10.1163/156856897X00366

Penton-Voak, I. S., Edwards, H., Percival, A., \& Wearden, J. H. (1996). Speeding up an internal clock in humans? Effects of click trains on subjective duration. Journal of Experimental Psychology: Animal Behavior Processes, 22, 307-320. doi:10.1037/00977403.22.3.307

Posner, M. I. (1980). Orienting of attention. The Quarterly Journal of Experimental Psychology, 32, 3-25. doi:10.1080/0033 5558008248231

Posner, M. I., \& Cohen, Y. (1984). Components of visual orienting. In H. Bouma \& D. G. Bouwhuis (Eds.), Attention and performance $X$ (pp. 531-556). Hillsdale: Erlbaum.

Posner, M. I., Nissen, M. J., \& Ogden, W. C. (1978). Attended and unattended processing modes: The role of set for spatial location. In H. L. Pick \& E. Saltzman (Eds.), Modes of perceiving and processing information (pp. 137-157). Hillsdale: Erlbaum.

Pratt, J., Hillis, J., \& Gold, J. M. (2001). The effect of the physical characteristics of cues and targets on facilitation and inhibition. Psychonomic Bulletin \& Review, 8, 489-495.

Rammsayer, T., \& Ulrich, R. (2005). No evidence for qualitative differences in the processing of short and long temporal intervals. Acta Psychologica, 120, 141-171. doi:10.1016/j.actpsy.2005. 03.005

Rolke, B., Ulrich, R., \& Bausenhart, K. M. (2006). Attention delays perceived stimulus offset. Vision Research, 46, 2926-2933. doi:10.1016/j.visres.2006.02.022

Schneider, K. A., \& Komlos, M. (2008). Attention biases decisions but does not alter appearance. Journal of Vision, 8, 1-10. doi:10.1167/8.15.3

Thomas, E. A., \& Brown, I. (1974). Time perception and the filledduration illusion. Perception \& Psychophysics, 16, 449-458.

Thomas, E. A., \& Cantor, N. E. (1975). On the duality of simultaneous time and size perception. Perception \& Psychophysics, 18, 44-48.

Thomas, E. A., \& Weaver, W. B. (1975). Cognitive processing and time perception. Perception \& Psychophysics, 17, 363-367.

Treisman, M. (1963). Temporal discrimination and the indifference interval: Implications for a model of the "Internal Clock". Psychological Monographs: General and Applied, 77, 1-31.
Treisman, M., Faulkner, A., Naish, P. L., \& Brogan, D. (1990). The internal clock: Evidence for a temporal oscillator underlying time perception with some estimates of its characteristic frequency. Perception, 19, 705-743. doi:10.10 68/p190705

Tse, P. U., Intriligator, J., Rivest, J., \& Cavanagh, P. (2004). Attention and the subjective expansion of time. Perception \& Psychophysics, 66, 1171-1189.

Ulrich, R. (2010). DLs in reminder and 2AFC tasks: Data and models. Attention, Perception, \& Psychophysics, 72, 1179-1198. doi:10.3758/APP.72.4.1179

Ulrich, R., Nitschke, J., \& Rammsayer, T. (2006). Perceived duration of expected and unexpected stimuli. Psychological Research, 70, 77-87. doi:10.1007/s00426-004-0195-4

Ulrich, R., Wing, A. M., \& Rinkenauer, G. (1995). Amplitude and duration scaling of brief isometric force pulses. Journal of Experimental Psychology: Human Perception and Performance, 21, 1457-1472. doi:10.1037/0096-1523.21.6.1457

Wearden, J. H. (1991). Do humans possess an internal clock with scalar timing properties? Learning and Motivation, 22, 59-83. doi:10.1016/0023-9690(91)90017-3

Wearden, J. H. (2004). Decision processes in models of timing. Acta Neurobiologiae Experimentalis, 64, 303-317.

Wearden, J. H., Edwards, H., Fakhri, M., \& Percival, A. (1998). Why 'sounds are judged longer than lights': Application of a model of the internal clock in humans. The Quarterly Journal of Experimental Psychology, 51B, 97-120.

Woodrow, H. (1951). Time perception. In S. Steven (Ed.), Handbook of experimental psychology (pp. 1224-1236). New York: Wiley.

Xuan, B., Zhang, D., He, S., \& Chen, X. (2007). Larger stimuli are judged to last longer. Journal of Vision, 7, 1-5. doi:10.1167/ 7.10 .2

Yeshurun, Y., \& Marom, G. (2008). Transient spatial attention and the perceived duration of brief visual events. Visual Cognition, 16, 826-848. doi:10.1080/13506280701588022

Zakay, D. (1993). Time estimation methods: Do they influence prospective duration estimates? Perception, 22, 91-101. doi:10.1068/ p220091

Zakay, D., \& Block, R. A. (1997). Temporal cognition. Current Directions in Psychological Science, 6, 12-16. doi:10.1111/14678721.ep11512604 